\title{
A Possible Correlation between Mass Ratio and Period Ratio in Multiple Planetary Systems
}

\author{
Tsevi Mazeh ${ }^{1}$ and Shay Zucker ${ }^{2}$ \\ mazeh@wise.tau.ac.il; shay@wise.tau.ac.il
}

\begin{abstract}
We report on a possible correlation between the mass ratio and period ratio of pairs of adjacent planets in extra-solar planetary systems. Monte-Carlo simulations show that the effect is significant to level of $0.7 \%$, as long as we exclude two pairs of planets whose periods are at the 1:2 resonance. Only the next few multiple systems can tell if the correlation is real.
\end{abstract}

Subject headings: planetary systems - solar system: general — stars: individual(GJ 876, HD 82943) — stars: statistics

\section{The correlation between the mass ratio and the period ratio}

As of March 2003, 101 extra-solar giant planets have been discovered, with minimum masses between 0.12 and 15 Jupiter masses and orbital periods between 2.986 and 5360 days $^{3}$. The periods and the masses of the planets are apparently correlated. The emerging population has shown indications for a correlation that corresponds to a paucity of massive planets with short orbital periods. Furthermore, this correlation does not appear in the population of planets that have been found in stellar binary systems (Zucker \& Mazeh 2002).

The known extra-solar planets include a special subgroup of 22 planets found in 10 multiple systems (Fischer et al. 2003), two of which consist of 3 planets ( $v$ And and 55 Cnc).

\footnotetext{
${ }^{1}$ School of Physics and Astronomy, Raymond and Beverly Sackler Faculty of Exact Sciences, Tel Aviv University, Tel Aviv, Israel

${ }^{2}$ Department of Geophysics and Planetary Sciences, Raymond and Beverly Sackler Faculty of Exact Sciences, Tel Aviv University, Tel Aviv, Israel

${ }^{3}$ see http://exoplanets.org/almanacframe.html
} 
In this Letter we focus on the masses and orbital periods of the planets found in the multiple systems, and present a distinctive correlation that characterizes this subsample.

The multiple systems provide us with a unique feature - the mass and period ratios between planets in adjacent orbits, ratios that can reflect general characteristics of the multiple systems. Although only the minimum masses are known for each planet, we assume the orbital inclinations of the planets in the same multiple system are similar, and therefore the ratio of the minumum masses is very close to the ratio of the actual masses. We therefore studied here the correlation between the (minimum) mass and the period ratios of all pairs of planets in adjacent orbits.

For each multiple system with two planets we derived one mass ratio and one orbitalperiod ratio. For each of the two systems with three known planets, we derived two sets of ratios - one set of ratios between the intermediate planet and the innermost one, and one set of ratios between the outermost and the intermediate one. Altogether, we have 12 such pairs of extra-solar planets. In Figure 1 we plotted their mass ratios as a function of their period ratios.

The Solar System includes two giant planets, Jupiter and Saturn, within the mass range of the known extra solar planets. We added an open circle in the Figure to represent the mass and period ratios of the Saturn/Jupiter pair. The mass and period ratios are very similar to those of $47 \mathrm{UMa}$, as already pointed out by Fischer et al. (2002).

The Figure shows an intriguing correlation between the two ratios. Except for two points that lie exactly at the 1:2 orbital-period resonance, all points seem to fit a straight line in a log-log plot. Considering the extra-solar planets alone, the correlation between the logarithms of the two ratios is 0.9415 and the best-fit line, which is also plotted in the Figure, has a slope of $0.92 \pm 0.10$ - suspiciously close to unity. When the fit includes the solar-system point, the correlation rises to 0.9498 .

\section{Significance}

The number of points in Figure 1 is extremely small. We have altogether only 12 points (excluding Saturn/Jupiter), out of which, we claim, 2 points should be excluded, because of their unique period ratio. On top of that, the data are subject to a strong selection effect that thwarts the detection of extra-solar planets with small masses and long periods. On the other hand, the correlation is intriguingly high, even for such a small number of points.

To estimate the significance of our findings we performed two randomization tests. In 
the first one we have used the masses and orbital periods of all known planets, a set that is supposedly subject to similar observational selection effect. We chose at random 8 pairs and 2 triples of orbits from the 101 extra-solar orbits, and calculated the correlation between their mass ratios and period ratios. We removed the two pairs that maximized the correlation for the remaining 10 pairs. Out of 1,000,000 random choices, only 7921 (0.8\%) yielded correlations higher than that obtained for the true data of the extra-solar pairs of planets.

The Monte-Carlo simulation described above shows the significance of the linear relation for the extra-solar planets alone. As we have seen in the previous section, the Saturn/Jupiter pair seems to agree with the same rule. In order to take account of this fact, we repeated the simulations, this time adding this pair to the randomly drawn 10 pairs and 2 triples, before rejecting two points. The significance implied by these modified simulations is $0.3 \%$ !

Note, however, that the selection effects of the single planets could have been quite different from the selection effects of the multiple systems. This might be the case mainly because once a planet has been discovered in a system, the observation strategy changes, leading to a different set of biases when additional planets are discovered in the same system. In general, large spread of a few radial velocity measurements of the stars in the sample is the first hint of the first planet, which usually entails further extensive series of observations. Large residuals relative to the derived orbit are a sign for a second planet, which can be the drive for additional observations that might detect and measure the orbital motion of the second (and third) planet.

Furthermore, the difficulty of extracting multiple signatures from the same data set probably introduces certain biases when looking for low mass planets in multiple systems. It might be more difficult to identify a low mass planet in a system which already contains a large planet if the orbital periods are similar rather than if they differ by a factor of 10 or more.

To try and overcome these difficulties we ran another randomization test in which we considered only the planets found in multiple systems. Again, we chose at random 8 pairs and 2 triples of orbits only from the 22 extra-solar orbits found at the multiple systems, and calculated the correlation between their mass ratios and period ratios. The period and mass distributions of the inner and outer planet in our simulated population are very similar to those of the actual pairs. For each set of simulated pairs we removed the two pairs that maximized the correlation for the remaining 10 pairs. Out of 1,000,000 random choices, only $6543(0.65 \%)$ yielded correlations higher than that obtained for the true data of the extra-solar pairs of planets. In order to visualize the distribution of the simulated correlation coefficients, we applied the Fisher's z-transformation (e.g., Kendall \& Stuart 1973) to the simulated coefficients, resulting in an approximately symmetrical distribution. The result is 
depicted in Figure 2. The correlation obtained for the true data is marked by an arrow.

Note that our simulations rejected the points whose removal optimally improved the correlation. We therefore expect the resulting correlation to be relatively high. In the true data the rejected points shared the property of lying exactly on the 1:2 resonance. We therefore feel our claim for a relatively high significance is well established.

Obviously, the reality of the correlation can be proved only by additional multiple systems, hopefully to be found in the near future.

\section{Discussion}

Figure 1 shows that multiple systems with a large period ratio (and therefore large orbital-radius ratio) show also a large ratio between their masses, with the more massive planet on the outside. Within the migration paradigm (e.g., Goldreich \& Tremaine 1980; Lin, Bodenheimer \& Richardson 1996), the present orbital radii of the planets are substantially smaller than the distance of their formation sites from their parent stars. Therefore the correlation we found, if verified, could be the result of some correlation between the migration range of a planet and it's mass. Massive planets might migrate slower (e.g., Ward 1997; Trilling et al. 1998; Nelson et al. 2000) and therefore are left far away when the disc evaporates. A similar effect could have caused the paucity of the massive planets with short periods (Zucker \& Mazeh 2002). If this is true, any point that represents a pair of planets in our parameter space slides to the right during migration, when the period of the smaller planet gets shorter.

Planets that find themselves in a 1:2 orbital resonance interact strongly with each other. The interaction could keep the period ratio stable against different migration rates that tend to push the smaller planet further in (e.g., Snellgrove, Papaloizou \& Nelson 2001). Therefore we suggest that if the migration scenario is responsible for the correlation we find, the pairs of planets with the 1:2 resonance did not succumb to this process, and therefore stayed at this resonance despite their large mass ratios.

The above general considerations do not explain why the correlation we found holds for more than two decades, and in particular why the exponent in the power law is so close to unity. Any theory of planetary formation and/or migration would have to explain these findings, if verified.

One other possibility to interpret the possible correlation is to assume the present periods reflect the original distances of the formation sites of the planets from their parent stars. 
Thus, the correlation we found may be related to a correlation between the location of the formation site of a planet and its mass. After all, the larger the disc radius at the formation site, the more mass is available for planetary accretion within a certain fraction of the planet original radius.

Interestingly enough, a similar idea has been proposed already by Laskar (2000), at a paper titled "On the Spacing of Planetary Systems". In that paper, he suggested a power-law relation

$$
\frac{m_{1}}{m_{2}}=\left(\frac{a_{1}}{a_{2}}\right)^{(2 p+3) / 6}
$$

which translates into a power-law relation between the mass and the period ratios. However, Laskar theory differs from the present data in two crucial details. Laskar considered cases where $p$ is between 0 and $-3 / 2$, which translates into an exponent between $1 / 3$ and 0 for the mass ratio - period ratio relation. The present data suggest an exponent which is close to unity. Furthermore, extension of Laskar relation goes, naturally, through the $\left(m_{2} / m_{1}, p_{2} / p_{1}\right)=(1,1)$ point, whereas ours does not. Therefore, Laskar theory by itself cannot explain the possible relation we suggest here, and we probably need a combination of models for formation and migration.

The present paper suffers from two drawbacks. First, although we have shown the effect seems to be statistically significant, the number of points in Figure 1 is still quite small. A few additional planetary systems are critically needed in order to establish the reality of the effect. Second, the authors do not claim to fully understand the possible correlation. If the effect is established in the future, we will need theoretical studies to understand the mechanism behind it.

We wish to thank M. Holman and the referee, J. Chambers, for important comments and advice. This work was supported by the Israeli Science Foundation (grant no. 40/00). S.Z. is grateful for partial support from the Jacob and Riva Damm Foundation.

\section{REFERENCES}

Fischer, D. A., Marcy, G. W., Butler, R. P., Laughlin, G., Vogt, \& S. S. 2002, ApJ, 564, 1028

Fischer, D. A., et al. 2003, ApJ, 586, 1394

Goldreich, P., \& Tremaine, S. 1980, ApJ, 241, 425

Kendall, M. C., \& Stuart, A. 1973, The Advanced Theory of Statistics, 3rd Edition (London: Charles Griffin \& Co.) 
Laskar, J. 2000, Phys. Rev. Lett., 84, 3240

Lin, D. N. C., Bodenheimer, P., \& Richardson, D. C. 1996, Nature, 380, 606

Nelson, R. P., Papaloizou, J. C. B., Masset, F., \& Kley, W. 2000, MNRAS, 318, 18

Snellgrove, M. D., Papaloizou, J. C. B., \& Nelson, R. P. 2001, A\&A, 374, 1092

Trilling, D. E., Benz, W., Guillot, T., Lunine, J. I., Hubbard, W. B., \& Burrows, A. 1998, ApJ, 500, 428

Ward, W. R. 1997, Icarus, 126, 261

Zucker, S., \& Mazeh, T. 2002, ApJ, 568, L113 


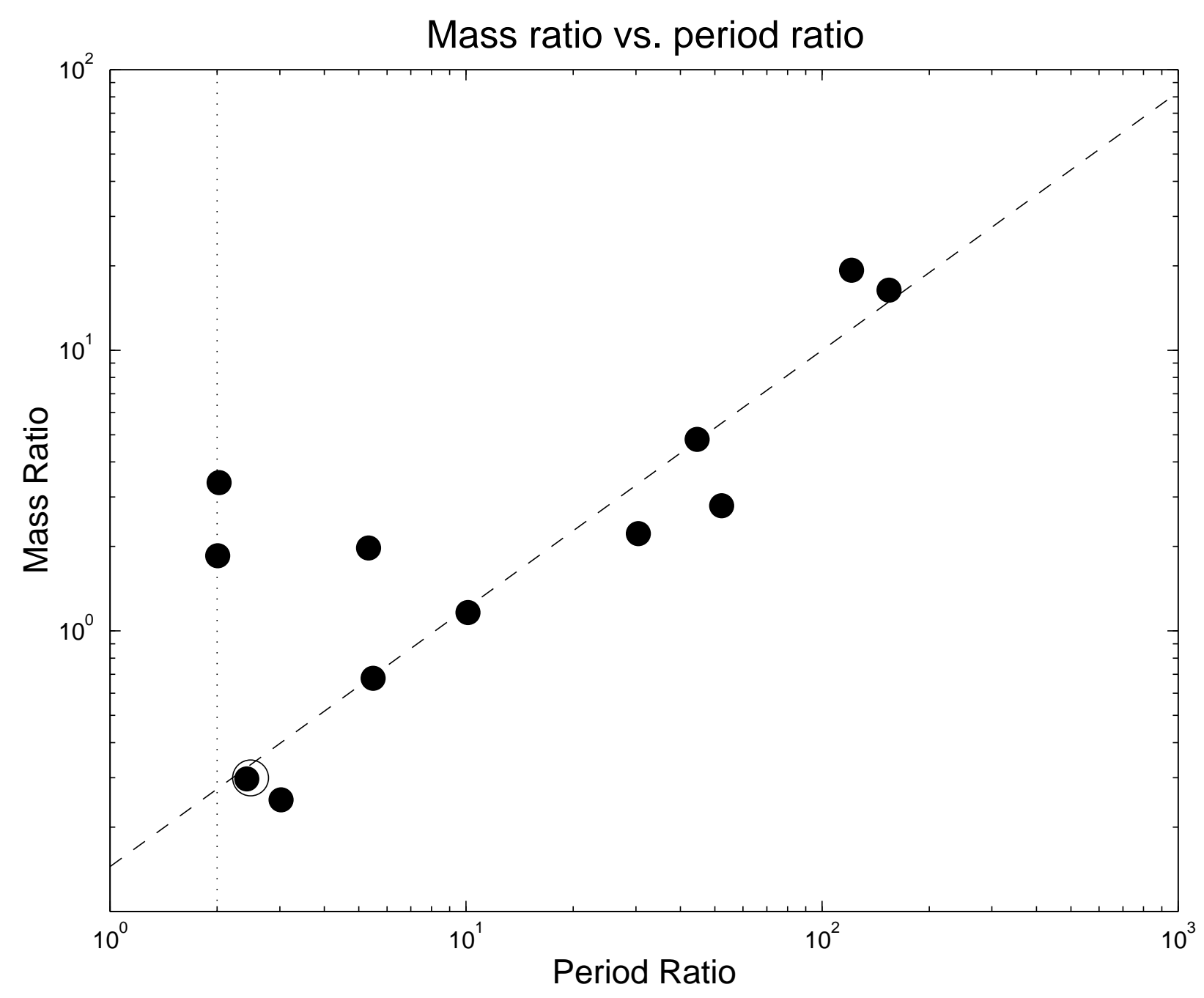

Fig. 1.- The mass and period ratios of the 12 extra-solar adjacent planet pairs. The open circle represents the Saturn/Jupiter pair. The dashed line represents the best-fit linear relation for the extra-solar planets, not including Saturn/Jupiter point. The vertical dotted line represents the 1:2 resonance. 


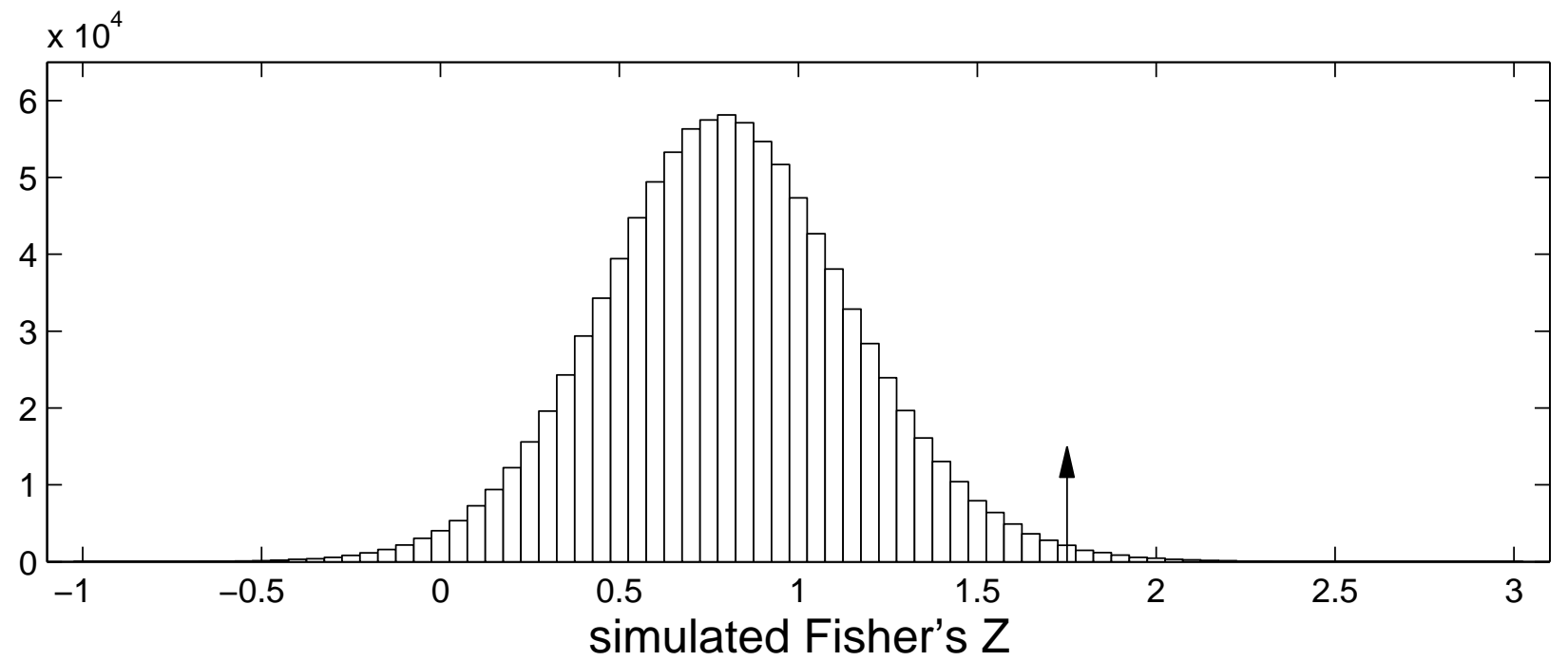

Fig. 2.- The distribution of $10^{6}$ simulated correlation coefficients (see text for details of the simulation). The correlation coefficients were transformed using the Fisher's ztransformation. The arrow corresponds to the correlation coefficient of the true data. 